\title{
Resistance of uropathogens to antibacterial agents: Emerging threats, trends and treatments
}

\author{
Gianpaolo Perletti ${ }^{1,2}$, Vittorio Magri ${ }^{3}$, Tommaso Cai ${ }^{4}$, Konstantinos Stamatiou ${ }^{5}$, \\ Alberto Trinchieri ${ }^{6}$, Emanuele Montanari ${ }^{7}$ \\ ${ }^{1}$ Department of Biotechnology and Life Sciences, Section of Medical and Surgical Sciences, Università degli Studi dell'Insubria, \\ Varese, Italy; \\ 2 Faculty of Medicine and Medical Sciences, Ghent University, Ghent, Belgium; \\ ${ }^{3}$ Urology Secondary Care Clinic, ASST-Nord, Milan, Italy; \\ ${ }^{4}$ Department of Urology, Santa Chiara Regional Hospital, Trento, Italy; \\ ${ }^{5}$ Department of Urology, Tzaneio General Hospital of Piraeus, Piraeus, Greece; \\ ${ }^{6}$ Urology Unit, A. Manzoni Hospital, Lecco, Italy; \\ ${ }^{7}$ Department of Urology, University of Milan - Ca' Granda Foundation - Ospedale Maggiore Policlinico, Milan, Italy.
}

\begin{abstract}
Summary Urinary tract infections are among the most common infectious diseases in humans. Today, resistance to nearly all antimicrobial classes is dramatically growing, and extremely drug-resistant or even pan-drug resistant pathogens are increasingly isolated around the world. It is foreseen that in the next decades the world will be facing a major medical emergency generated by the rapid spread of pathogens carrying resistance determinants of unprecedented power. Carbapenemase-producing Enterobacteriaceae, multidrug-resistant Enterococci and fluoroquinolone resistance determinants in both Gram-negative and Gram-positive uropathogens are among the greatest emergencies. In this article, the major emerging threats of particular interest to urologists are reviewed, worldwide resistance trends are illustrated, and novel and older - but still active - recommended drugs are summarized.
\end{abstract}

KEY WORDS: Urinary tract infections; Resistance; Antibacterial agents; Antibiotics; Carbapenemase; Carbapenem resistance.

Submitted 11 April 2018; Accepted 11 April 2018

\section{INTRODUCTION}

Urinary tract infections (UTIs) are among the most common bacterial-borne diseases in humans. Although antibacterial agents have been the mainstay of treatment for UTIs for decades, today routine antibiotic therapy is seriously threatened by worldwide outbursts of infections involving multidrug-resistant (MDR), extremely drug-resistant (XDR) or even pan drug-resistant pathogens. Gramnegative Enterobacteriaceae like Klebsiella pneumoniae (KP) and Escherichia coli (EC), and Gram-positive cocci like Enterococcus faecalis and Enterococcus faecium are at the same time the most common uropathogens and the bacteria carrying the most powerful resistance determinants. On the basis of a general assessment of the major resistance threats, the present review will focus on worldwide susceptibility trends resulting from large international surveillance studies, and will present some available therapeutic options -either novel or old but still effective- for the management of resistant infections of urological interest.

\section{EMERGING RESISTANCE THREATS}

In 2013, the Centers for Disease Control and Prevention (USA) included carbapenem-resistant Enterobacteriaceae (CREB) among the three microorganisms posing an urgent threat to public health, due to the extensive resistance shown by these pathogens to a wide array of antibacterial agents, and to the very high mortality rates reported in patients with bloodstream infections caused by organisms like carbapenem-resistant KP.

Several international programs are now aimed at fostering research, development and awareness about the threat of pathogen resistance. Pivotal European trials are for example EURECA (European prospective cohort study on Enterobacteriaeae Showing Resistance to Carbapenems), and REVISIT (Revisiting serious bacterial infection with innovation), promoted in the frame of the European COMBACTE-CARE project, an initiative of the combacte.com collaboration.

\section{Carbapenem resistance}

For several decades, extended-spectrum beta-lactamases (ESBL) of TEM and SHV lineage, conferring resistance to a variety of penicillins and third-generation cephalosporins, have represented a worrisome problem for physicians treating patients affected by infectious diseases. In these cases, carbapenem therapy was a useful therapeutic resource against ESBL-producing pathogens. However, today the usefulness of carbapenems is decreasing rapidly, and the global spread of carbapenemase-producing Enterobacteriaceae (the etiological agents of lung, soft tissue and urinary tract infections) has undoubtedly become the most important threat in the field of infectious diseases. The speed of such spread is alarming: for example, whereas in 2009 the spread of the Klebsiella pneumoniae carbapenemase (KPC) in the USA was classified as 'sporadic' in half of the federal states and 'less than sporadic' in the remaining states (1), in 2014 the entire USA territory has been defined as affected by an 'endemic' presence of KPC (2).

The concern raised by this mounting trend is due to the 
fact that the spectrum of enzymatic activity of carbapenemases is not restricted to drugs like imipenem, ertapenem, meropenem or doripenem, but extends to almost all beta-lactams. Moreover, resistance to carbapenems and other beta-lactams is in most cases facilitated by horizontal-transfer of genetic elements like transposons and plasmid-borne integrons, invariably containing multiple resistance determinants which often confer multidrugresistant, extensively drug-resistant or even pan drug-resistant properties to pathogens.

$K$. pneumoniae is the most prevalent pathogen among carbapenem-resistant Enterobacteriaceae according to a recent surveillance report by the European Centre for Disease Prevention and Control (3). Infection with carbapenemase-producing KP (CPKP) can double the mortality rate of affected patients (from $21 \%$ to $42 \%$, all infections), and can triplicate the mortality of non-intensive care patients with UTIs from $13 \%$ to $43 \%$ in intensive care unit (ICU) cases (4).

The carbapenemases that have been characterized so far belong to all four Ambler classes of beta-lactamases (Table 1). Among the enzymes of concern for urologists, the class-A, plasmid-borne K. pneumoniae serine carbapenemase (KPC) is today the most common transmissible resistance determinant in Enterobacteriaceae. KPC includes more than 20 variants (the most prevalent being KPC-2 and -3), who can hydrolyze virtually all beta-lactam agents including carbapenems, penicillins, broad spectrum cephalosporins and monobactams. KPCs are only weakly inhibited by clavulanate and tazobactam $(1,5)$, and the encoding blaKPC gene, predominantly present in IncF plasmids (with FIIK replicons, and often associated with a Tn4401 trasposon-like structure), is very often coexpressed with cotrimoxazole, fluoroquinolone and aminoglycoside resistance determinants (6).

Notably, today KPC is no longer exclusively expressed in KP (mainly the ST258 strain) or other CREB, but is also found in other species such as Pseudomonas aeruginosa (7). The SME enzyme also belongs to

Table 1.
Carbapenem resistance determinants in pathogens involved in UTIs.

\begin{tabular}{|c|c|c|c|c|c|c|}
\hline 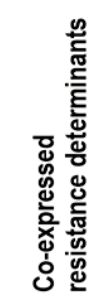 & 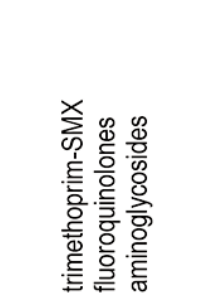 & & 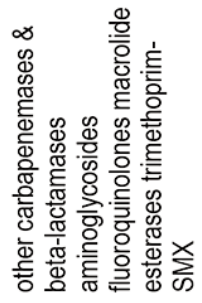 & - & - & - \\
\hline 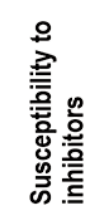 & 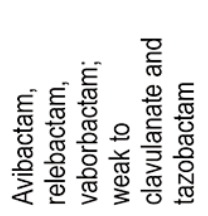 & & 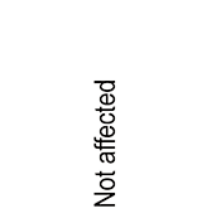 & 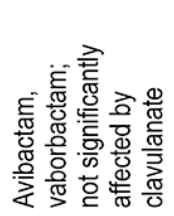 & 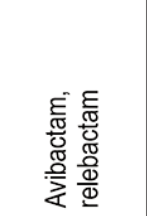 & - \\
\hline 吾 & 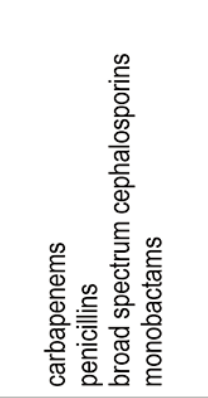 & & 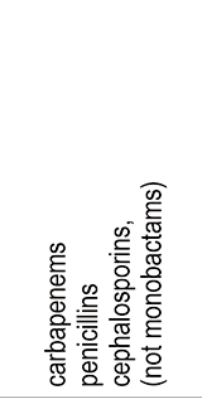 & 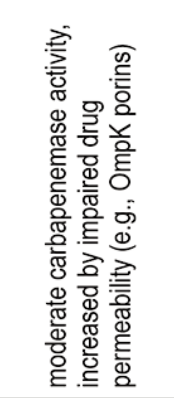 & 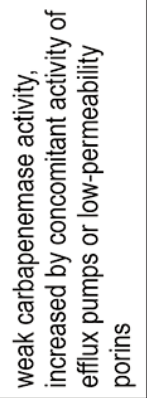 & - \\
\hline 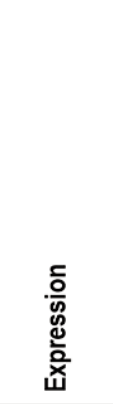 & 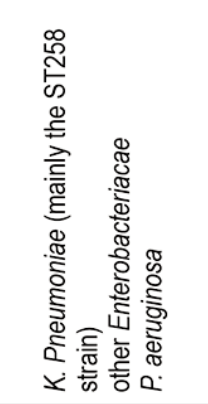 & 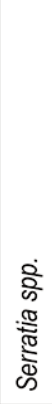 & 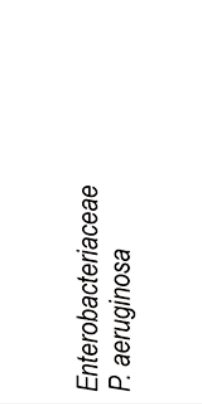 & 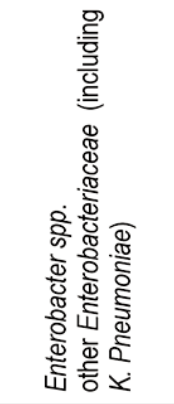 & 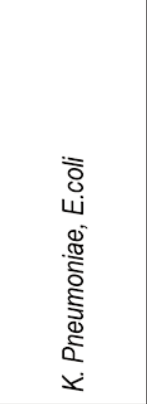 & 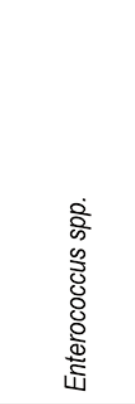 \\
\hline 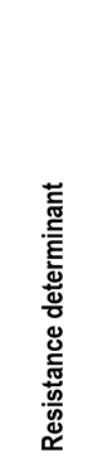 & 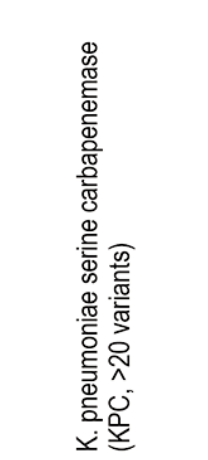 & 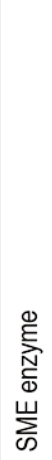 & 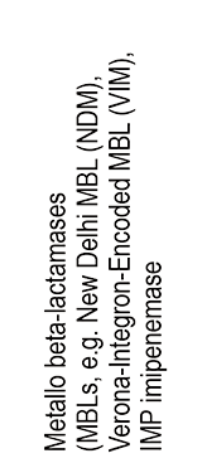 & 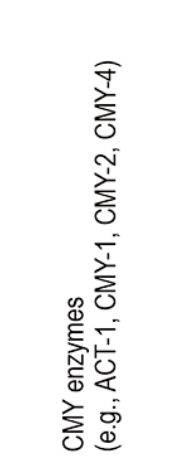 & 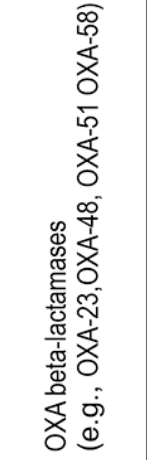 & 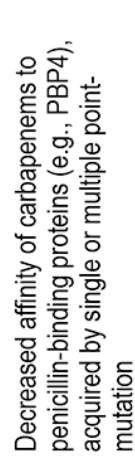 \\
\hline 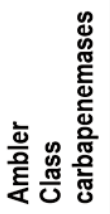 & $\ll$ & & $\varpi$ & 0 & 口 & 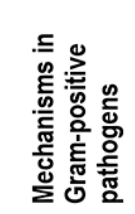 \\
\hline
\end{tabular}

class-A carbapenemases, but at present is isolated less frequently than KPC, being in almost all cases restricted 
to the Serratia marcescens species, a member of the Enterobacteriaceae family (8).

Class B metallo beta-lactamases/carbapenemases (MBLs) require zinc for their catalytic activity. These enzymes are expressed in Enterobacteriaceae but also in P. aeruginosa, and can hydrolyze carbapenems, penicillins and cephalosporins, but not monobactams. Importantly, MBLs are not affected by beta-lactamase inhibitors.

The most powerful and most diffuse MBLs are the New Delhi MBL (NDM), the Verona-Integron-Encoded MBL (VIM) and the IMP imipenemase (9). The blaNDM gene variants are harbored by a variety of broad-host-range Inc plasmids like IncA/C, IncF, IncR, IncN, IncM, IncX, which are in turn expressed within various pathogen isolates, including the highly prevalent ST11 KP clone. Pathogens harboring the NDM are often residually susceptible to a single last-resort agent like polymixin E, since NDM is very frequently co-expressed with other carbapenemases (OXA-48, VIM and/or KPC) and with multiple resistance determinants such as ESBL, AmpC beta-lactamases, aminoglycoside-modifying enzymes, fluoroquinolone resistance enzymes (e.g., Qnr), macrolide esterases, as well as determinants conferring resistance to trimethoprim-sulphametoxazole.

Among class $C$ beta-lactamases, the CMY enzymes (e.g., ACT-1, CMY-1, CMY-2, and CMY-4, often transferred via pYMG plasmids) were originally expressed in Enterobacter spp. but now are being carried by several other Enterobacteriaceae, including KP (CMY-10). ACT-1 and CMY-1 show moderate carbapenemase activity, are not significantly inhibited by clavulanic acid, and highlevel resistance to carbapenems is often the result of combined drug hydrolysis and impaired drug permeability (e.g., involving OmpK35/36 porins) (10, 11).

Class D OXA beta-lactamases can efficiently hydrolyze penicillins like oxacillin. Some OXA enzymes, such as OXA-23, OXA-48, OXA-51 and OXA-58, carried mainly by highly transferable IncL group plasmids like pOXA48a, have a weak carbapenemase activity. Nevertheless, in pathogens like ST-11 KP, high-MIC carbapenem resistance may result from the concomitant activity of efflux pumps or low-permeability porins (12).

As far as carbapenem-resistance in Gram-positive pathogens is concerned, three mechanisms of reduced beta-lactam susceptibility have been reported in Enterococci: (i) beta-lactamase production as well as (ii) overproduction or (iii) inactivation (by point-mutation) of penicillin-binding proteins like PBP4 or PBP5. Notably, carbapenem resistance is mainly caused by point-mutations in PBP4. For example, resistance to faropenem in E. faecalis is due to decreased affinity of the drug for PBP4, due to the acquisition of one or two point mutations in the PBP4-encoding gene (13).

\section{Plasmid-mediated fluoroquinolone resistance}

Plasmid mediated fluoroquinolone resistance (PMQR) is increasing to the point that international guidelines no longer recommend drugs like ciprofloxacin, ofloxacin or levofloxacin as first-choice agents for treatment of urinary tract infections, when resistance is assessed in at least $10 \%$ of pathogen isolates $(14,15)$.

Fluoroquinolones (FQ) inhibit the activity of bacterial gyrase and topoisomerase IV in Gram-negative and -positive pathogens. Mutations occurring in the quinolone resistance determining regions of genes encoding these type II topoisomerases (e.g., in gyrA, gyrB, parC or parE subunits) are the most common chromosomal determinants of FQ resistance (16). For example, in Enterococci resistance to fluoroquinolones is mainly caused by mutations in the GyrA and ParC genes in gyrase and topoisomerase IV, respectively (17). Other mechanisms of resistance to $F Q$ are the qepA-encoded efflux pumps, the OqxAB-encoded efflux pumps -very common in plasmids carried by $\mathrm{K}$. penumoniae-, the qnr-encoded proteins, impeding the interaction of FQ with DNA gyrase, and a mutant aminoglycosyde acetyl-transferase (aac (6')-Ib-cr) which acetylates the piperazine ring of FQ like norfloxacin and ciprofloxacin (18-21).

Determinants of PMQR are horizontally transferable. For example, qnrA1, A3, A6, B2, B4, B6 and B10 are associated with the mobilizing element insertion sequence ISCR, whereas qnrBl and B20 are associated with IS26 and Orf1005. The aac (6')-Ib-cr enzyme-encoding gene is often found in association with qnrB and blaCTX in a cassette within an IS26 transposon. In addition, qepA and OqxAB are also often mobilized by IS26 transposons (22).

\section{Polymyxin resistance}

Polymyxins (polymyxin B and colistin/polymyxin E) are antibiotics produced by the Gram-positive species Paenibacillus polymyxa. The plasmidic mor-1 colistin resistance gene, expressed in Klebsiella spp. and other Enterobacteriaceae, encodes for a phosphatidylethanolamine transferase enzyme, lessening the affinity of colistin towards the lipid-A on bacterial cell membranes via enzymatic modification (23). This resistance determinant, rapidly spreading worldwide (24), represents a major threat since polymyxins are considered last-resource antibiotics against CREB.

\section{Glycopeptide resistance}

Together with Enterobacteriaceae, Enterococci are the most common etiological determinants of urinary tract infections. Acquired resistance to glycopeptide antibiotics in Enterococci is given by five gene clusters: vanA, vanB, vanD, vanE and vanG, leading to the expression of peptidoglycal pentapeptide precursors (PPP) characterized by poor affinity for vancomycin and other glycopeptides. Interestingly, vanA Enterococci are resistant to the lastgeneration glycopeptide dalbavancin but are susceptible to oritavancin, possibily due to the unique dual mechanism of action of the latter (25). Horizontal transfer of Van genes may occur via Tn-1546 transposons, which are found in conjugative and non-conjugative plasmids (26).

\section{WORLDWIDE RESISTANCE TRENDS}

\section{Beta-lactam antibiotic resistance}

Information about worldwide trends in pathogen resistance is not frequently published, due to the fact that global epidemiological studies are difficult to perform, and most studies focus on a single nation or region. Nevertheless, quality works like for example the 2016 
systematic review by Lee et al. (27), or some global surveillance studies, which are occasionally performed, give access to evidence concerning global trends of pathogen chemoresistance.

The Study to Monitor Antimicrobial Resistance Trends (SMART) is among the largest global surveillance programs aimed at monitoring longitudinal antimicrobial resistance patterns worldwide. Over 200,000 clinical samples have been collected since 2002 from patients with complicated intra-abdominal infections, whereas isolates from patients with UTIs have been acquired since 2009. Among other surveillance actions, susceptibility testing to 12 commonly used antibacterial agents has been performed in different regions of the world. One-hundred ninety-four hospital sites in countries located within the macro-regions of Asia/Pacific, Latin America, Middle East/Africa, North America, and Europe have taken part in the project. Within the SMART program, the ESKAPE group of pathogens (Enterococcus spp, Staphylococcus aureus, Klebsiella pneumoniae, Acinetobacter baumannii, Pseudomonas aeruginosa and Enterobacter spp.) is of main interest to urologists and accounts for the vast majority of resistance encountered in nosocomial settings.

Global trends. Data from the SMART project provide an excellent overview of global resistance trends for uropathogens. In the frame of SMART, the 2009-2010 susceptibility analysis of E. coli isolates from UTI specimens of hospitalized patients in countries worldwide showed an overall $17.9 \%$ prevalence of extended-spectrum beta lactamase (ESBL) resistance determinants (28), whose rate varied depending on the region considered (Asia/Pacific, 27.7\%, Latin America, 23.3\%, Europe, 18.8\%, Middle East/Africa, 16.2\%, Northern America, 7.4\%).

Only imipenem and ertapenem demonstrated > 90\% susceptibility in ESBL-positive E. coli at that time (99.7\% and $98 \%$ isolates susceptible, respectively), whereas susceptibility to amikacin and piperacillin-tazobactam was lower (87.1\% and $84.4 \%$, respectively). The least active agents were ampicillin-sulbactam, all cephalosporins except cefoxitin, and the fluoroquinolones (28). In the discussion of their data, Hoban et al. emphasized the relevance of intermediate susceptibility in the treatment of UTIs, as certain antibacterial agents concentrate physiologically in the urine (28). This suggests that in specific infections showing intermediate susceptibility towards certain agents, high-dose treatment may be attempted in the absence of more suitable options.

As far as the geographic distribution of carbapenemases is concerned, the overall mortality due to CREB reported in studies from Northern America, Southern America, Europe, and Asia was 33.2\%, 46.7\%, 50\%, and 44.8\%, respectively (all infections) (4).

KP carbapenemases (KPC) are endemic in the USA, China, Greece, Italy, Poland, Israel, Brazil, Argentina, Colombia and Taiwan, whereas sporadic spread of KPC-producing KP has been observed in virtually all Asian, European and American countries (summarized in: 27).

The New Delhi metallo beta-lactamases (NDM) confer resistance to most beta-lactams including carbapenems, as well as co-resistance to aminoglycosides, tetracyclines, fluoroquinolones and other antibacterials, due to the comp- resence of various resistance determinants within the same mobile genetic elements. NDM are endemic India, Pakistan and Bangladesh (27). An analysis of the prevalence of NDM in the in the frame of the SMART program for years 2008 2012 - published in 2015 - showed that isolates of nine different species of NDM-expressing Enterobacteriaceae were disseminated in India, Serbia, the Philippines, Saudi Arabia, Guatemala, Vietnam and Georgia, and that different variants of the NDM gene were compresent with CTX-M cephalosporinases (29). Moreover, Enterobacteriaceae producing the VIM and IMP carbapenemases (alone or coexpressed) have been found in Greece, Italy, Spain, the Philippines, Turkey, Australia, Mexico, USA and India, with most isolates concomitantly nonsusceptible to one or more agents like ampicillin-sulbactam, ceftriaxone, cefepime, ceftazidime, piperacillin-tazobactam, ciprofloxacin, amikacin, tigecycline and colistin (30). Importantly, KP isolates produced IMP-26 together with various VIM variants (VIM-1, 5, 26 or 27), whereas E. coli expressed IMP-1 alone (30). Class-D OXA carbapenemases are endemic in India, Turkey, Morocco, Libya, Egypt and Tunisia, and sporadic spreads have been documented throughout Europe and the Middle-East (summarized in: 27). OXA enzymes have been recently isolated in all continents.

Asia-Pacific. In the Asia-Pacific region, 60 centers from 31 countries provided susceptibility data, which were collected in years 2010-2013 and published in 2016 (31). Uropathogenic E. coli (UPEC) and KP were the most prevalent isolates. China showed the highest rates of ESBL, both in UPEC and KP $(66.4 \%$ in 2010 and $59.9 \%$ in 2013 for UPEC; $60 \%$ in 2010 and $54.5 \%$ in 2013 for KP). Besides China, dramatic increases of ESBL-expressing UPEC were observed for example in Thailand (54.2\% in 2013 vs. $36.8 \%$ in 2010) and Hong Kong (41.7\% in 2013 vs. $25 \%$ in 2010), while ESBL-KP were substantially increased in New Zeland (45.5\% in 2013 vs. $23.1 \%$ in 2010), South Korea (55.6\% in 2013 vs. $37.5 \%$ in 2010) and the Philippines (61.3\% in 2013 vs. $37.9 \%$ in 2010). In the same region, Jean et al. reported a significant increase of the annual rates of carbapenemase prevalence between year $2008(0.07 \%)$ and year 2014 (1.1\%), with the blaNDM-1 allele becoming more prevalent than the IMP-26 gene (32).

Latin America. In 11 Latin American countries, analysis of resistance trends in years 2013-2015, published in 2017, showed that ESBL-positive KP accounted for $46.6 \%$ of UTI isolates, substantially higher than the values assessed in SMART 2003 (14\%). ESBL-positive rates of KP were higher $(52.9 \%)$ in isolates from intensive care unit (ICU) compared to non-ICU patients (44.4\%) (33). Ertapenem and imipenem showed slightly lower rates of resistance, compared to other beta-lactams $(37.4 \%$ and $24.1 \%$, respectively, versus, e.g., > 38\% for cephalosporins and piperacillin-tazobactam). Among other antimicrobials tested, the aminoglycoside amikacin continues to show high rates of susceptibility (92.3\%) for isolates of KP (33). Northern America. The SMART program provided 24655 isolates from Europe and North America, obtained from ICU (17.8\%) and NON-ICU (82.2\%) wards. Data from 
90 hospitals in Canada, in the USA and in 18 European countries were collected. By comparing these regions, in 2015 Lob and coworkers found that resistance among Enterobacteriaceae in Europe was largely driven by KP expressing high rates of ESBLs (41.2\% in intensive care units; mostly CTX-M) and carbapenemases (13.2\%; mostly KPC and OXA-types). For all Enterobacteriaceae combined, only ertapenem and amikacin inhibited > $90 \%$ of ICU isolates in both regions. In Northern America, ertapenem, imipenem and amikacin inhibited $>90 \%$ of KP, whereas in Europe only amikacin attained such levels, according to Lob et al. (34).

In 2016, the same research group also focused on susceptibility patterns of ESBL in E. coli from UTIs, representing the 52\% of Gram-negative pathogens collected. A significant increase in ESBL prevalence was seen in the US (from $7.8 \%$ in 2010 to $18.3 \%$ in 2014 , $\mathrm{P}<0.0001$ ), whereas in Canada an increase from 10.4\% to $13.0 \%$ was not statistically significant (35). Moreover, isolates from hospital-acquired UTIs increased considerably in the US (9.4\% in 2010 to $27.7 \%$ in 2014). The steepest increases over the five-year study period were found among US males (from $7.1 \%$ to $26.2 \%$ ) and older US patients (from $8.8 \%$ to $26.6 \%)(34)$.

Europe. National surveillance programs worldwide have reported extensive spreads of carbapenemase-producing uropathogens. Logan and Weinstein have edited maps showing the spread of CREB throughout Europe and the world (6). According to the 2017 survey published by the European Centre for Disease Prevention and Control (ECDC), $25 \%$ to $50 \%$ isolates of KP are carbapenemresistant in Italy, Romania and Greece, the latter showing rates beyond the upper limit (36). In Europe, the diffusion of KPC is 'endemic' in Greece, Poland and Italy (1). From a study involving 3324 non-replicate isolates of Enterobacteriaceae from Italian hospitals, it was found that $4.3 \%$ of isolates were non-susceptible to carbapenems, K. pneumoniae being the most prevalent carrier of the blaKPC carbapenemase gene (in 25.1\% and 7.7\% inpatients and outpatients, respectively) (37).

A recent ECDC report focusing on Italy, pointed to dramatically increasing resistance trends in this country. For example, the proportion of CPKP blood isolates increased from $1.3 \%$ in 2006 to $33.5 \%$ in 2015 , whereas combined resistance to third-generation cephalosporins, fluoroquinolones and aminoglycosides increased from $2.8 \%$ in 2005 to $29.7 \%$ in 2015 . However, in E. coli the proportion of carbapenem-resistant blood isolates remained low: $0.1 \%$ in 2007 to $0.2 \%$ in 2015 , though combined resistance increased from $0.8 \%$ in 2002 to $14.6 \%$ in 2015 (38). In Germany, a very low incidence of carbapenemase-producing isolates has been documented, as a multicenter study published in 2016 demonstrated an incidence of 0.047 cases per 1000 hospital admissions (39). Low prevalence has also been reported in Belgium, with 3.5\% of Enterobacteriaceae being carbapenem non-susceptible through expression of OXA-48, OXA-427, KPC and NDM (40). A steep growth of carbapenem resistance has been reported in France. The OXA-48 and NDM resistance determinants increased from $23.1 \%$ in 2012 to $36.2 \%$ in 2014 (41).

\section{Non-beta-lactam antibiotic resistance}

As far as non-beta-lactam antibiotic susceptibility trends are concerned, certain African countries show dramatic prevalences of pan-resistant infections. For example, it has been reported that in Nigeria uropathogenic E. coli (UPEC) is pan-resistant to cotrimoxazole (100\% isolates), and highly resistant to ofloxacin (70\%), gentamycin (92\%) and tetracycline (88\%) (42).

In various Asia-Pacific countries, the SMART program reported that the rates of susceptibility to levofloxacin among hospital isolates of E. coli ranged from $83 \%$ in New Zeland, to $39 \%$ in Singapore, to 15\% in India (cited in: 43). The overall prevalence of fluoroquinolone resistance in UPEC is dramatically increasing worldwide, with increasing trends in Brazil $(63.53 \%$ increase for ciprofloxacin and $66.50 \%$ for norfloxacin from 2010 to 2015)(44), and with values up to 17\% in Italy and 38\% in Turkey (summarized in: 45), whereas in the US a 10year study reported an increase from 3\% to 17\% between 2000 and 2010 (46)

In Europe, the areas showing the highest rates of $\mathrm{PMQR}$ are the Mediterranean countries. This distribution extends to the whole Mediterranean basin, and the affected countries are Croatia, Slovenia, Greece, Italy, Spain, France, Morocco, Algeria, Tunisia, Egypt, and Turkey (47, 48). Resistance to cotrimoxazole in community-acquired UPEC is high in Southern America (64\% in Nicaragua and up to $58 \%$ in pediatric cases in Brazil), Turkey (up to 43\%) and Greece (27.3\%) whereas in the USA a percentage of 24.2 has been reported (45)

The prevalence of vancomycin-resistant Enterococci (VRE) causing symptomatic or asymptomatic infections in Europe ranges between 1 and 30\%, whereas in the USA, VRE are about the $30 \%$ of all nosocomial isolates (49). Encouragingly, Toner et al. reported that the proportion of VRE has remained stable between 2006 (13.9\%) and $2014(11.5 \%)$, whereas a worrisome increase of resistance to nitrofurantoin has been documented in frame of the same study (just above zero in 2005 to over $40 \%$ in 2009 to about $20 \%$ in 2014). Notably, in that study the prevalence of vancomycin resistance was substantially higher in E. faecium (51.2\%) compared to E. faecalis $(1.6 \%)(49)$

Despite the data mentioned above, uropathogens worldwide seem to retain sensitivity against agents like nitrofurantoin and fosfomycin. For example, the prevalence of resistance to nitrofurantoin in UPEC isolates has been reported to be $2.9 \%$ in Brazil (year 2007, 50), <2\% in Europe and 1.6 in the US $(45,49,50)$. The figures concerning fosfomycin appear to be as low, with a prevalence in several European countries inferior to 2\% $(51,52)$.

As far as infections of urological concern in the pediatric population are concerned, resistance to ciprofloxacin in E. coli UTIs increased 10-fold between 2002 and 2009 both in young boys and girls, as shown in a study performed in 195 US pediatric hospitals (1\% to $10 \%$ and $0.6 \%$ to $4 \%$ of isolates, respectively) (53).

\section{AvaILABLE AND EMERgING THERAPEUtic STRATEgIES}

Few randomized controlled studies concerning novel or improved therapeutic protocols against drug-resistant 
infections have been performed so far. Thus, the available evidence is mainly observational and sometimes limited to case reports. In most cases, studies focusing on Enterobacteriaceae are based on complicated cases of pneumonia or bacteremia. Despite these limitations, this section will focus on selected therapeutic options -novel or old but still efficacious- for treatment of infections caused by resistant pathogens of concern to urologists.

\section{Carbapenems}

It is known that certain carbapenemase enzymes can decrease the susceptibility of pathogens to carbapenems to a limited extent, and PK/PD data suggest that T > MIC targets can be met with high probability of attainment with high-dose continuous infusions of carbapenems (e.g. 6 g/day meropenem) when MICs are ranging between 4 and $16 \mathrm{mg} / \mathrm{L}$ (summarized in: 54).

These experimental data are encouraging, though experts suggest that monotherapy with these agents is not advisable, and that combination therapy including a carbapenem (when MICs are $\leq 8 \mathrm{mg} / \mathrm{L}$ ) can result in lower mortality rates (54). importantly, the efficacy of combined therapies including a carbapenem appears to be MIC-dependent, as mortality rates in sepsis patients infected with KPCproducing KP were up to $35 \%$ for MICs $>16 \mathrm{mg} / \mathrm{L}$, but as low as $13.3 \%$ if the MICs of meropenem $(2 \mathrm{~g}$, $>3 \mathrm{~h}$ infusion thrice daily) were below or equal to $4 \mathrm{mg} / \mathrm{L}$ (55).

Interestingly, combination of two carbapenems might become a last-resort regimen for treating pandrug-resistant and colistin-resort KPC-producing KP infections (bacteremia, pneumonia, and UTIs). The rationale for such approach is based on the fact that KPC appears to have higher affinity for ertapenem compared to other carbapenems. Thus, while KPC would be "engaged" by ertapenem, a co-administered different carbapenem could exert its bactericidal activity. Although recent quality observational data are encouraging (56-58), additional, adequately powered studies are urgently warranted. Moreover, such evidence may ideally foster the development of ertapenem analogues characterized by higher affinity for KPC compared to the founder compound, in order to optimally exploit such "engagement" activity on carbapenemases.

\section{Aztreonam}

Monobactam therapy may be considered as an option against Enterobacteriaceae expressing class B or D carbapenemases when these determinants are not coexpressed with class A enzymes, which are able to efficiently hydrolyze aztreonam (59). In all cases, susceptibility testing is necessary, due to variable monobactamase activity shown by different subclasses of class-B enzymes.

\section{Avibactam}

The novel non-beta-lactam beta-lactamase inhibitor avibactam (Figure 1) has been approved in 2016 in the European Union for treatment of soft tissue infections, pneumonia and urinary tract infections in combination with the third-generation cephalosporin ceftazidime. Avibactam inhibits a broad spectrum of beta-lactamases including A-class, C-class and some D-class carbapene- mases (60). In vitro assessments performed in the frame of the INFORM global surveillance study demonstrated that $98 \%$ of CREB isolates containing KPC or OXA-48 enzymes were susceptible to this combination, even when the isolates expressed ESBLs or AmpC enzymes $(61,62)$. However, ceftazidime/avibactam was ineffective against carbapenem-hydrolyzing metallo-beta-lactamases like NDM.

In vitro activity of this combination was also demonstrated in the frame of a phase 3 trial involving ceftazidime-resistant UTIs (63). Recently, Jayol et al. investigated the in vitro activity of ceftazidime/avibactam, alone (for class A and D carbapenemase producers) or in combination with aztreonam (for class-B carbapenemase

Figure 1.

Chemical structure of the novel non-beta-lactam beta-lactamase inhibitors avibactam, relebactam and vaborbactam. Structures were drawn using the PubChem $\mathrm{NIH}$ public repository database (93).
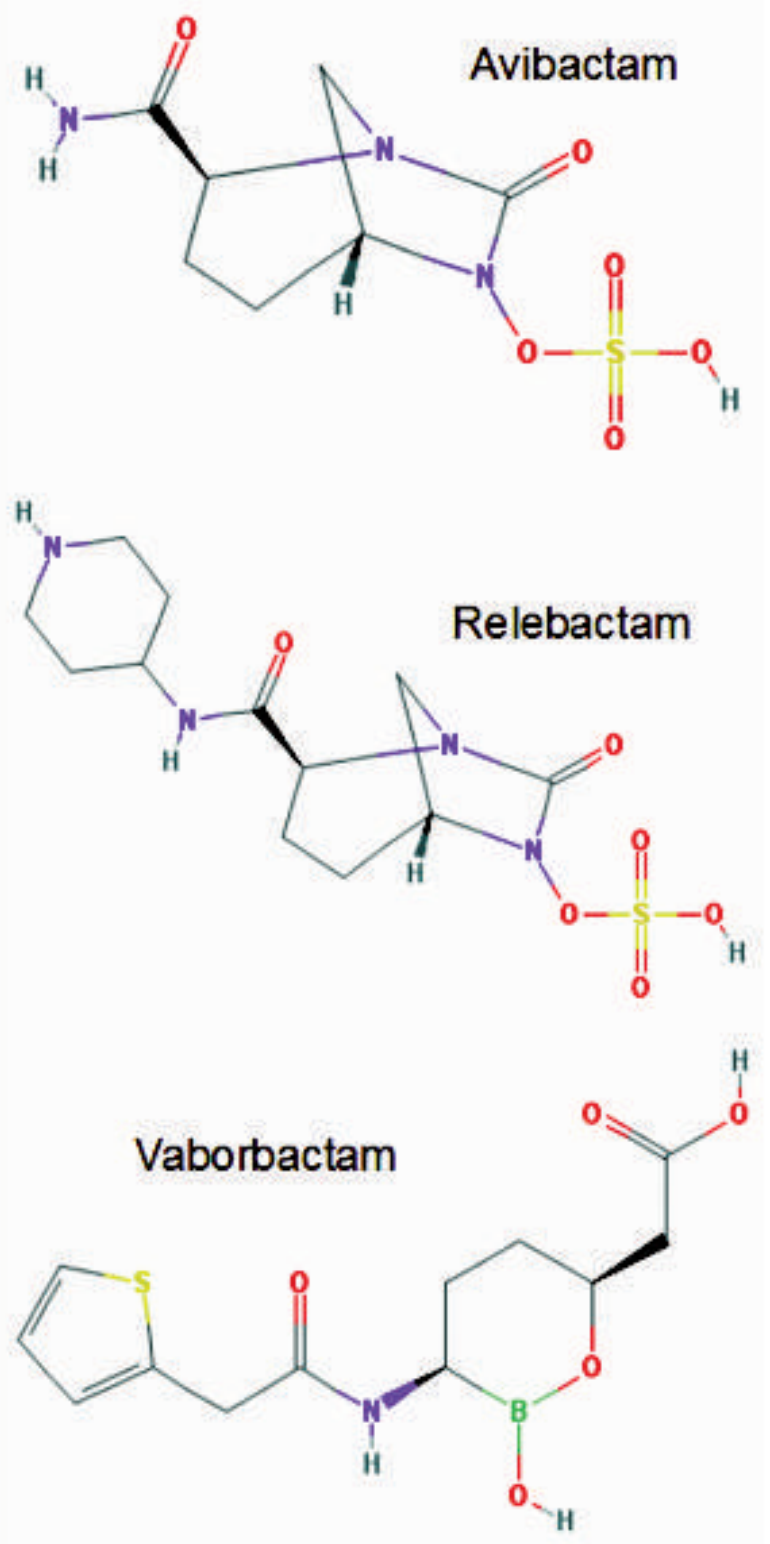
producers), against a collection of colistin-resistant and carbapenemase-producing KP isolates (64).

It was shown that ceftazidime/avibactam was effective against colistin-resistant and KPC-producing or OXA48-producing KP, and was also efficient against KP isolates co-expressing two carbapenemases. Interestingly, the combination of ceftazidime/avibactam with aztreonam was synergic against NDM-producing KP.

Investigators suggested that such synergic activity could be explained by the neutralization of the ESBL activity by avibactam, which can in turn restore the susceptibility of KP to aztreonam (64). These data warrant urgent clinical investigation

The clinical efficacy of the ceftazidime/avibactam combination $(2 \mathrm{~g} / 0.5 \mathrm{~g}$, intravenous $\mathrm{q} 8 \mathrm{~h})$ was demonstrated by recent phase 3 studies in patients with complicated UTIs, including acute pyelonephritis $(65,66)$. Further studies are urgently needed to investigate the clinical cure rates of this combination in UTIs caused by CREB.

\section{Relebactam}

The SMART program provided isolates from the USA, which were used for testing the activity of imipenem combined with the newly developed, renally excreted carbapenemase inhibitor relebactam. Relebactam is structurally related to avibactam, differing only by the presence of an amide functional group bound to a piperidine ring (Figure 1). For KP, 99\% and $96.1 \%$ of isolates were susceptible to imipenem-relebactam and imipenem alone, respectively, and $74.1 \%$ of imipenem-resistant isolates were rendered susceptible to the carbapenem by addition of relebactam (67). In vitro assays showed that the combination imipenem/relebactam decreased the MICs of KPC-producing KP compared to imipenem alone (MIC50: 0.25/4 mg/L, MIC90: 1/4 mg/L), but was not as effective against pathogens expressing class $\mathrm{D}$ enzymes (e.g., OXA-48 in KP) (68). To date, phase II clinical trials have reported that imipenem/relebactam is as effective as imipenem alone for treatment of complicated UTIs, including acute pyelonephritis (69).

Imipenem-relebactam is currently investigated in the frame of phase III clinical trials for the treatment of imipenem-resistant infections.

\section{Vaborbactam}

Vaborbactam (VB) is a boronic acid-based non-beta lactam beta-lactamase inhibitor, registered by FDA in 2017 for therapy of complicated urinary tract infections, combined with meropenem $(2 \mathrm{~g} / 2 \mathrm{~g}$, intravenous q8h, to be adjusted in patients with renal impairment). Its chemical structure is not related to the ones of avibactam or relebactam (Figure 1).

Similar to meropenem, VB is renally excreted, and up to $60 \%$ of a dose is found unchanged in the urine within 24 hours. VB inhibits class A beta-lactamases (including $\mathrm{KPC}$ ) and class $C$ AmpC $\beta$-lactamases.

The meropenem/VB combination decreases the MICs of most resistant Enterobacteriaceae (2-fold to > 1024-fold decrease), though it appears that the addition of VB does not improve the activity of meropenem against $P$. aeruginosa (70).

Meropenem/VB is active against CREB isolates and against Enterobacteriaceae showing multidrug-resistant and extensively drug-resistant phenotypes (MIC50/90: $0.5 / 32,0.03 / 1$, and $0.5 / 32 \mathrm{mg} / \mathrm{L}$, respectively), though this drug combination showed limited activity against isolates expressing metallo- $\beta$-lactamases (e.g., NDM- 1 , VIM) and oxacillinases (e.g., OXA-48, OXA-163) detected in Asia-Pacific and in certain European countries (71). The TANGO-1 phase III clinical trial has reported superiority of meropenem/VB over piperacillin/tazobactam for the treatment of complicated UTIs, including acute pyelonephritis (72). The TANGO-2 randomized, openlabel phase III clinical trial of meropenem/VB versus "best available therapy" in patients with complicated urinary tract infections, bacteremia or pneumonia, was stopped early due to a benefit-risk ratio in favor of meropen$\mathrm{em} / \mathrm{VB}$ (73).

\section{Polymyxins}

The polymyxin antibiotics colistin (poliymyxin E) and poliymyxin B have become a mainstay in the treatment of CREB infections. The EUCAST breakpoint for resistance is $2 \mathrm{mg} / \mathrm{L}$. Recent studies resulted in the recommendation to prescribe high doses of the antibiotic (up to 10 million international units), divided into twice- or thrice-daily administrations (74). Importantly, combination therapy including colistin and rifampin was shown to be effective against colistin-resistant, KPC-producing KP (75). Though new polymyxin derivatives with decreased toxicity are under development, nephrotoxicity occurring in about $40 \%$ of cases and neurotoxicity are limiting factors to the extensive administration of colistin for treatment of CREB-induced infections. A possible limiting factor to the full exploitation of polymyxins for UTIs may also be the limited renal clearance of these drugs, though pharmacokinetic studies seem to confirm that the urinary recovery of colistin may be sufficient to attain concentrations above the MICs shown for example by XDR P. aeruginosa (76).

\section{Aminoglycosides}

Plazomicin is a novel aminoglycoside ("neoglycoside") antibiotic closely related to sisomicin and structurally recalling gentamicin. Its molecular structure was designed to be resistant against aminoglycoside-modifying enzymes, which are often expressed in CREB isolates (77). For example, in non-NDM-expressing CREB, the MICs of plazomicin ranged between $>0.5$ and $2 \mathrm{mg} / \mathrm{L}$, compared to $0.25 />256 \mathrm{mg} / \mathrm{L}$ and $1 / 128 \mathrm{mg} / \mathrm{L}$ for gentamicin and amikacin, respectively (78).

Three-hundred multidrug resistant ESBL-producing and/or carbapenemase-producing Enterobacteriaceae isolates from Athens, Greece (a CREB endemic area), most of which were also resistant to previous generation aminoglycosides (e.g., $\mathrm{MIC}_{50} / \mathrm{MIC}_{90}$ to amikacin $=32 />32$ ), were tested for sensitivity to plazomicin. This novel aminoglycoside retained activity against all tested isolates of K. pneumoniae, E. coli, and Enterobacter spp., with $\mathrm{MIC}_{50}$ and $\mathrm{MIC}_{90}$ of 1 and $2 \mu \mathrm{g} / \mathrm{ml}$, respectively, irrespective of their multidrug-resistant phenotype (79). In strains of CREB resistant to gentamicin, tobramycin and amikacin, plazomicin exhibited an MIC range of 0.12-4 $\mathrm{mg} / \mathrm{L}$, with $\mathrm{MIC}_{50}$ and $\mathrm{MIC}_{90}$ values of 0.25 and $1 \mathrm{mg} / \mathrm{L}$, 
respectively. Interestingly, in CPKP isolates, synergy was observed when plazomicin was combined with meropenem, colistin or fosfomycin, whereas the combination with tigecycline resulted in indifference (80).

The safety and efficacy of plazomicin vs. levofloxacin in treating UTIs was assessed in a phase II comparative study, which ended in 2012 (81). Microbiological eradication in the plazomicin group $(15 \mathrm{mg} / \mathrm{kg})$ was achieved in $93.1 \%$ of the patients whose baseline urinary pathogens had a plazomicin MIC of $4 \mathrm{mg} / \mathrm{L}$.

Microbiological eradication in the group receiving levofloxacin $(750 \mathrm{mg}$ ) was achieved in $93.8 \%(15 / 16)$ of patients with levofloxacin-susceptible baseline urinary pathogens $(\mathrm{MIC}=4 \mathrm{mg} / \mathrm{L})$. The EPIC and CARE phase III trials, investigating the safety and efficacy of plazomicin in patients with complicated UTIs and other infections caused by gram-negative pathogens including CREB, have been concluded but not yet published in the form of journal articles.

\section{Fosfomycin}

Only case reports are available showing the efficacy of fosfomycin -administered in monotherapy for uncomplicated UTIs or combined with colistin against urosepsisin infections caused by Enterobacteriaceae expressing KPC, OXA-48 and NDM carbapenemases $(82,83)$. Case reports include accounts of successful combination therapy including dual carbapenem plus oral fosfomycin in UTIs caused by NDM-expressing Enterobacteriaceae (57). Such approach is preferable, as the rapid development of resistance during therapy is a major problem related to the usage of fosfomycin as single agent (84).

Intravenous formulations of fosfomycin allow administration of high doses of the drug (e.g., $4 \mathrm{~g} q 6 \mathrm{~h}$ ), if necessary (85). A small prospective case series including eleven critically ill ICU patients affected by CPKP bacteremia, UTI or pneumonia, was based on administration of intravenous fosfomycin (2-4g q6h) for about 14 days, combined with colistin $(n=6)$, gentamicin $(n=3)$ or piperacillin/tazobactam $(n=1)$, based on ascertained susceptibility. All-cause hospital mortality was $18.2 \%$, and no infection relapse was observed in enrolled patients. Intravenous therapy with high-dose fosfomycin appeared to be well tolerated, without renal or liver function test abnormalities (86). Further large-scale studies are warranted to confirm these encouraging safety and efficacy results.

\section{Oritavancin}

Oritavancin is a semi-synthetic lipoglycopeptide, characterized by a threefold mechanism of action: (i) inhibition of the transglycosylation pathway in the bacterial wall synthesis, (ii) inhibition of the transpeptidation step by binding to the peptide bridging cell wall segments and (iii) cell wall disruption.

A recent in vitro subset study in the frame of a prevalence analysis performed on over 140.000 bloodstream isolates of Enterococci from Europe and the USA demonstrated that oritavancin was active against vancomycin-resistant $E$. faecalis (MIC50/90, 0.25-0.5 mg/L), and showed $\mathrm{MIC}_{50}$, $\mathrm{MIC}_{90}$ and $\mathrm{MIC}_{100}$ values of $0.03,0.12$ and $0.25 \mathrm{mg} / \mathrm{L}$ against VanA-positive E. faecium, respectively (87).
Oritavancin is renally and fecally excreted as unmodified molecule and may represent an interesting agent for treating complicated UTIs, though such approach would be off-label, as this drug is approved for the moment only for Gram-positive skin and skin structure infections.

\section{Fluoroquinolones}

Chen et al. have examined in detail how the pharmacokinetic-pharmacodynamic properties of fluoroquinolones could be exploited to design treatment strategies in areas with high rates of fluoroquinolone resistance (43). Levofloxacin appears to be a better option compared to ciprofloxacin, since the excretion of the former is by $87 \%$ renal (ciprofloxacin: 50\%), and since efflux pumps like AcrAB, MdfA and NorE are more active on the latter (88). Thus, as the bactericidal activity of these agents is concentration-dependent, the achievement of high peak urine concentrations as a result of high-dose treatment with levofloxacin may be the key for eradicating pathogens showing intermediate FQ resistance levels. For example, a single $750 \mathrm{mg}$ dose of levofloxacin achieves a mean urinary $C_{\max }$ of $620 \mathrm{mg} / \mathrm{L}$, with prolonged post-antibiotic effect, compared to $340 \mathrm{mg} / \mathrm{L}$ attained by a standard $500 \mathrm{mg}$ dose (89). Chen et al. also suggested that in areas with resistance uropathogen rates $>20 \%$, high-dose levofloxacin might be considered as an option for UTIs caused by E. coli isolates showing a MIC $\leq 32 \mathrm{mg} / \mathrm{L}$, after taking into consideration the potential adverse effects of such therapy (43).

The aac (6')-I gene product (AAC) confers to Enterobacteriaceae resistance to aminoglycoside antibiotics through acetylation of specific - $\mathrm{NH} 2$ residues at the level of specific amino sugars. A mutated variant of AAC (aac (6')-Ib-cr) confers resistance to certain fluoroquinolones via acetylation of the $(=\mathrm{NH})$ residue within the piperazine ring of drugs like ciprofloxacin or norfloxacin. This decreases by 4 -fold the susceptibility of pathogens to such drugs (90) and increases by 16 times the fluoroquinolone mutant prevention concentration in E. coli $(0.2 \rightarrow 3.2 \mathrm{mg} / \mathrm{L})$, thus facilitating the survival of target site mutants (91). Interestingly, FQs like levofloxacin have a methylated piperazine residue which is virtually protected from the action of AAC. Thus, if resistance rates caused by the AAC are present or suspected, levofloxacin, or other "protected" FQs like prulifloxacin or pefloxacin may be temptatively administered.

\section{Glycylcyclines}

Together with colistin, tigecycline is often the only agent to which CPKP is residually susceptible. However, the use of this agent for treatment of urological infections is significantly associated with subsequent development of resistance, as shown by van Duin and coworkers (OR, 6.13; 95\% CI, 1.15-48.65) (92).

\section{Conclusions}

In conclusion, in the next decades the world will be facing a major medical emergency generated by the rapid spread of pathogens carrying resistance determinants of unprecedented power. All medical specialties will be affected by such spread, including foremostly urology. 
As far as urinary tract diseases are concerned, we believe that the old definition of complicated vs uncomplicated infections should be modified, as any UTI involving carbapenemase-expressing uropathogens, or MDR/XDR uropathogens, should be considered and managed as complicated conditions.

Urgent containment measures must be put into effect, with priority given to those areas of the world characterized by climatic conditions favoring the seasonal outburst of infection epidemics, but also by negligent clinical practice, unprofessional pharmaceutical dispensing, as well as by poor patient compliance and education. Rigorous antibiotic stewardship and restriction of novel and old last resort agents to the sole hospital setting will also contribute to the containment of "superbug" epidemics.

Regretfully, major pharmaceutical companies have abandoned research for novel antibacterial agents due to the little financial reward ensured by drugs which can cure diseases in a very short time and at the same time may become rapidly obsolete due to the emerging of pathogen resistance.

Nevertheless, public or private research mainly aimed at discovering new therapies against Gram-negative organisms is of vital importance. Only the future will tell us who will prevail in the struggle for survival between humans and bacteria, and ultimately in the war between the immense resources of human ingenuity and the immense adaptability of genomes and species.

\section{ACKNOWLEDGEMENTS}

We are thankful to medical student Louise Beckers (Ghent University School of Medicine) for assistance in literature search and retrieval, and in study data extraction.

\section{REFERENCES}

1. Nordmann P, Cuzon G, Naas T. The real threat of Klebsiella pneumoniae carbapenemase-producing bacteria. Lancet Infect Dis. 2009; 9:228-36.

2. Nordmann P, Poirel L. The difficult-to-control spread of carbapenemase producers among Enterobacteriaceae worldwide. Clin Microbiol Infect. 2014; 20:821-30.

3. https://ecdc.europa.eu/sites/portal/files/documents/AMR-surveillance-Europe-2016.pdf.

4. Xu L, Sun X, MaX. Systematic review and meta-analysis of mortality of patients infected with carbapenem-resistant Klebsiella pneumoniae. Ann Clin Microbiol Antimicrob. 2017; 16:18.

5. Patel G, Bonomo RA. "Stormy waters ahead": global emergence of carbapenemases. Front Microbiol. 2013; 4:48.

6. Logan LK, Weinstein RA. The Epidemiology of CarbapenemResistant Enterobacteriaceae: The Impact and Evolution of a Global Menace. J Infect Dis. 2017; 215:S28-S36.

7. Villegas MV, Lolans K, Correa A, et al. First identification of Pseudomonas aeruginosa isolates producing a KPC-type carbapenem-hydrolyzing beta-lactamase. Antimicrob Agents Chemother. 2007; 51:1553-5.

8. Hopkins KL, Findlay J, Meunier D, et al. Serratia marcescens producing SME carbapenemases: an emerging resistance problem in the UK? J Antimicrob Chemother. 2017; 72:1535-1537.
9. Bushnell G, Mitrani-Gold F, Mundy LM. Emergence of New Delhi metallo- $\beta$-lactamase type 1-producing Enterobacteriaceae and non-enterobacteriaceae: global case detection and bacterial surveillance. Int J Infect Dis. 2013; 17:e325-33.

10. Cao VT, Arlet G, Ericsson BM, et al. Emergence of imipenem resistance in Klebsiella pneumoniae owing to combination of plasmid-mediated CMY-4 and permeability alteration. J Antimicrob Chemother. 2000; 46:895-900.

11. Stapleton PD, Shannon KP, French GL. Carbapenem resistance in Escherichia coli associated with plasmid-determined CMY-4 betalactamase production and loss of an outer membrane protein. Antimicrob Agents Chemother. 1999; 43:1206-10.

12. Walther-Rasmussen J, Høiby N. OXA-type carbapenemases. J Antimicrob Chemother. 2006; 57:373-83.

13. Hiraga N, Muratani T, Naito S, et al. Genetic analysis of faropenem-resistant Enterococcus faecalis in urinary isolates. J Antibiot (Tokyo). 2008; 61:213-21.

14. Gupta K, Hooton TM, Naber KG, et al. International clinical practice guidelines for the treatment of acute uncomplicated cystitis and pyelonephritis in women: A 2010 update by the Infectious Diseases Society of America and the European Society for Microbiology and Infectious Diseases. Clin Infect Dis. 2011; 52:e103-20.

15. http://uroweb.org/guideline/urological-infections/\#3.

16. Yoshida H, Bogaki M, Nakamura M, et al. Quinolone resistancedetermining region in the DNA gyrase gyrA gene of Escherichia coli. Antimicrob Agents Chemother. 1990; 34:1271-25.

17. Korten V, Huang WM, Murray BE. Analysis by PCR and direct DNA sequencing of gyrA mutations associated with fluoroquinolone resistance in Enterococcus faecalis. Antimicrob Agents Chemother. 1994; 38:2091-4

18. Tran JH, Jacoby GA, Hooper DC. Interaction of the plasmidencoded quinolone resistance protein Qnr with Escherichia coli DNA gyrase. Antimicrob Agents Chemother. 2005; 49:118-25.

19. Robicsek A, Strahilevitz J, Jacoby GA, et al. Fluoroquinolonemodifying enzyme: a new adaptation of a common aminoglycoside acetyltransferase. Nat Med. 2006; 12:83-8.

20. Yamane K, Wachino J, Suzuki S, et al. New plasmid-mediated fluoroquinolone efflux pump, QepA, found in an Escherichia coli clinical isolate. Antimicrob Agents Chemother. 2007; 51:3354-60.

21. Hansen LH, Johannesen E, Burmølle M, et al. Plasmid-encoded multidrug efflux pump conferring resistance to olaquindox in Escherichia coli. Antimicrob Agents Chemother. 2004; 48:3332-7.

22. Ruiz E, Sáenz Y, Zarazaga M, et al. qnr, aac(6')-Ib-cr and qepA genes in Escherichia coli and Klebsiella spp.: genetic environments and plasmid and chromosomal location. J Antimicrob Chemother. 2012; 67:886-97.

23. Gao R, Hu Y, Li Z, et al. Dissemination and Mechanism for the MCR-1 Colistin Resistance. PLoS Pathog. 2016; 12:e1005957.

24. Jeannot K, Bolard A, Plésiat P. Resistance to polymyxins in Gram negative organisms. Int J Antimicrob Agents. 2017; 49:526-535.

25. Woodford N. Biological counterstrike: antibiotic resistance mechanisms of Gram-positive cocci. Clin Microbiol Infect. 2005; 11 Suppl 3:2-21.

26. Hollenbeck BL, Rice LB. Intrinsic and acquired resistance mechanisms in Enterococcus. Virulence. 2012; 3:421-33.

27. Lee CR, Lee JH, Park KS, et al. Global Dissemination of Carbapenemase-Producing Klebsiella pneumoniae: Epidemiology, 
Genetic Context, Treatment Options, and Detection Methods. Front Microbiol. 2016 Jun 13; 7:895.

28. Hoban DJ, Nicolle LE, Hawser S, et al. Antimicrobial susceptibility of global inpatient urinary tract isolates of Escherichia coli: results from the Study for Monitoring Antimicrobial Resistance Trends (SMART) program: 2009-2010. Diagn Microbiol Infect Dis. 2011; 70:507-11.

29. Biedenbach D, Bouchillon S, Hackel M, et al. Dissemination of NDM metallo- $\beta$-lactamase genes among clinical isolates of Enterobacteriaceae collected during the SMART global surveillance study from 2008 to 2012. Antimicrob Agents Chemother. 2015; 59:826-30.

30. Peirano G, Lascols C, Hackel M, et al. Molecular epidemiology of Enterobacteriaceae that produce VIMs and IMPs from the SMART surveillance program. Diagn Microbiol Infect Dis. 2014; 78:277-81.

31. Jean SS, Coombs G, Ling T, et al. Epidemiology and antimicrobial susceptibility profiles of pathogens causing urinary tract infections in the Asia-Pacific region: Results from the Study for Monitoring Antimicrobial Resistance Trends (SMART), 2010-2013. Int J Antimicrob Agents. 2016; 47:328-34.

32. Jean SS, Hsueh PR; SMART Asia-Pacific Group. Distribution of ESBLs, AmpC $\beta$-lactamases and carbapenemases among Enterobacteriaceae isolates causing intra-abdominal and urinary tract infections in the Asia-Pacific region during 2008-14: results from the Study for Monitoring Antimicrobial Resistance Trends (SMART). J Antimicrob Chemother. 2017; 72:166-171.

33. Karlowsky JA, Hoban DJ, Hackel MA, et al. Resistance among Gram-negative ESKAPE pathogens isolated from hospitalized patients with intra-abdominal and urinary tract infections in Latin American countries: SMART 2013-2015. Braz J Infect Dis. 2017; 21:343-348.

34. Lob SH, Biedenbach DJ, Badal RE, et al. Antimicrobial resistance and resistance mechanisms of Enterobacteriaceae in ICU and non-ICU wards in Europe and North America: SMART 2011-2013. J Glob Antimicrob Resist. 2015; 3:190-197.

35. Lob SH, Nicolle LE, Hoban DJ, et al. Susceptibility patterns and ESBL rates of Escherichia coli from urinary tract infections in Canada and the United States, SMART 2010-2014. Diagn Microbiol Infect Dis. 2016; 85:459-65.

36-https:/lecdc.europa.eu/sites/portal/files/documents/AMR\%202016_ Final-with-cover-for-web-2017.pdf

37. Giani T, Antonelli A, Caltagirone M, et al. Evolving beta-lactamase epidemiology in Enterobacteriaceae from Italian nationwide surveillance, October 2013: KPC-carbapenemase spreading among outpatients. Euro Surveill. 2017; 22.

38. https://ecdc.europa.eu/sites/portal/files/documents/AMR-country-visit-Italy.pdf

39. Kaase M, Schimanski S, Schiller R, et al. Multicentre investigation of carbapenemase-producing Escherichia coli and Klebsiella pneumoniae in German hospitals. Int J Med Microbiol. 2016; $306: 415-20$

40. De Laveleye M, Huang TD, Bogaerts $P$, et al. Increasing incidence of carbapenemase-producing Escherichia coli and Klebsiella pneumoniae in Belgian hospitals. Eur J Clin Microbiol Infect Dis. 2017; 36:139-146

41. Dortet L, Cuzon G, Ponties V, et al. Trends in carbapenemaseproducing Enterobacteriaceae, France, 2012 to 2014. Euro Surveill. $2017 ; 22$.
42. Okesola AO, Aroundegbe TI. Antibiotic resistance pattern of uropathogenic Escherichia coli in South West Nigeria. Afr J Med Med Sci. 2011; 40:235-8.

43. Chen $Y H, K o W C, H s u e h P R$. The role of fluoroquinolones in the management of urinary tract infections in areas with high rates of fluoroquinolone-resistant uropathogens. Eur J Clin Microbiol Infect Dis. 2012; 31:1699-704.

44. Rodrigues WF, Miguel CB, Nogueira AP, et al. Antibiotic Resistance of Bacteria Involved in Urinary Infections in Brazil: A Cross-Sectional and Retrospective Study. Int J Environ Res Public Health. 2016; 13

45. Nolan LK, Li G, Logue CM. Origin and Dissemination of Antimicrobial Resistance among Uropathogenic Escherichia coli. Microbiol Spectr. 2015; 3

46. Sanchez GV, Master RN, Karlowsky JA, et al. In vitro antimicrobial resistance of urinary Escherichia coli isolates among U.S. outpatients from 2000 to 2010. Antimicrob Agents Chemother. 2012; 56:2181-3

47. Yanat B, Rodríguez-Martínez JM, Touati A. Plasmid-mediated quinolone resistance in Enterobacteriaceae: a systematic review with a focus on Mediterranean countries. Eur J Clin Microbiol Infect Dis. 2017; 36:421-435

48. Stamatiou K, Pierris N. Mounting resistance of uropathogens to antimicrobial agents: A retrospective study in patients with chronic bacterial prostatitis relapse. Investig Clin Urol. 2017; 58:271-280.

49. Toner L, Papa N, Aliyu SH, et al. Vancomycin resistant enterococci in urine cultures: Antibiotic susceptibility trends over a decade at a tertiary hospital in the United Kingdom. Investig Clin Urol. $2016 ; 57: 129-34$

50. Kiffer CR, Mendes C, Oplustil CP, et al. Antibiotic resistance and trend of urinary pathogens in general outpatients from a major urban city. Int Braz J Urol. 2007; 33:42-8.

51. Kahlmeter G, Poulsen HO. Antimicrobial susceptibility of Escherichia coli from community-acquired urinary tract infections in Europe: the ECO.SENS study revisited. Int J Antimicrob Agents. 2012; 39:45-51.

52. Kresken $M$, Körber-Irrgang B, Biedenbach DJ, et al. Comparative in vitro activity of oral antimicrobial agents against Enterobacteriaceae from patients with community-acquired urinary tract infections in three European countries. Clin Microbiol Infect. 2016; 22:63.e1-63.e5.

53. Edlin RS, Shapiro DJ, Hersh AL, et al. Antibiotic resistance patterns of outpatient pediatric urinary tract infections. J Urol. 2013, 190:222-7.

54. Morrill, Thaden JT, Pogue JM, et al. Role of newer and reemerging older agents in the treatment of infections caused by carbapenem-resistant Enterobacteriaceae. Virulence. 2017; 8:403-416.

55. Tumbarello M, Viale P, Viscoli C, et al. Predictors of mortality in bloodstream infections caused by Klebsiella pneumoniae carbapenemase-producing $K$. pneumoniae: importance of combination therapy. Clin Infect Dis. 2012; 55:943-50.

56. Souli M, Karaiskos I, Masgala A, et al. Double-carbapenem combination as salvage therapy for untreatable infections by KPC2-producing Klebsiella pneumoniae. Eur J Clin Microbiol Infect Dis. 2017; 36:1305-1315

57. De Pascale G, Martucci G, Montini L, et al. Double carbapen$\mathrm{em}$ as a rescue strategy for the treatment of severe carbapenemaseproducing Klebsiella pneumoniae infections: a two-center, matched case-control study. Crit Care. 2017; 21:173. 
58. Rosa R, Rudin SD, Rojas LJ, et al. "Double carbapenem" and oral fosfomycin for the treatment of complicated urinary tract infections caused by bla(NDM) -harboring Enterobacteriaceae in kidney transplantation. Transpl Infect Dis. 2018; 20.

59. Morrill HJ, Pogue JM, Kaye KS, et al. Treatment Options for Carbapenem-Resistant Enterobacteriaceae Infections. Open Forum Infect Dis. 2015; 2:ofv050.

60. Lahiri SD, Johnstone MR, Ross PL, et al. Avibactam and class C $\beta$-lactamases: mechanism of inhibition, conservation of the binding pocket, and implications for resistance. Antimicrob Agents Chemother. 2014; 58:5704-13.

61. de Jonge BL, Karlowsky JA, Kazmierczak KM, et al. In Vitro Susceptibility to Ceftazidime-Avibactam of CarbapenemNonsusceptible Enterobacteriaceae Isolates Collected during the INFORM Global Surveillance Study (2012 to 2014). Antimicrob Agents Chemother. 2016; 60:3163-9

62. Karaiskos I, Giamarellou H. Multidrug-resistant and extensively drug-resistant Gram-negative pathogens: current and emerging therapeutic approaches. Expert Opin Pharmacother. 2014; 15:1351-70.

63. Stone GG, Bradford PA, Newell P, et al. In Vitro Activity of Ceftazidime-Avibactam against Isolates in a Phase 3 Open-Label Clinical Trial for Complicated Intra-Abdominal and Urinary Tract Infections Caused by Ceftazidime-Nonsusceptible Gram-Negative Pathogens. Antimicrob Agents Chemother. 2017; 61.

64. Jayol A, Nordmann P, Poirel L, et al. Ceftazidimelavibactam alone or in combination with aztreonam against colistin-resistant and carbapenemase-producing Klebsiella pneumoniae. J Antimicrob Chemother. 2018; 73:542-544.

65. Wagenlehner FM, Sobel JD, Newell $P$, et al. Ceftazidimeavibactam Versus Doripenem for the Treatment of Complicated Urinary Tract Infections, Including Acute Pyelonephritis: RECAPTURE, a Phase 3 Randomized Trial Program. Clin Infect Dis. 2016; 63:754-762.

66. Carmeli Y, Armstrong J, Laud PJ, et al. Ceftazidime-avibactam or best available therapy in patients with ceftazidime-resistant Enterobacteriaceae and Pseudomonas aeruginosa complicated urinary tract infections or complicated intra-abdominal infections (REPRISE): a randomised, pathogen-directed, phase 3 study. Lancet Infect Dis. 2016; 16:661-673.

67. Lob SH, Hackel MA, Kazmierczak KM, et al. In Vitro Activity of Imipenem-Relebactam against Gram-Negative ESKAPE Pathogens Isolated by Clinical Laboratories in the United States in 2015 (Results from the SMART Global Surveillance Program). Antimicrob Agents Chemother. 2017; 61.

68. Lapuebla A, Abdallah M, Olafisoye O, et al. Activity of Imipenem with Relebactam against Gram-Negative Pathogens from NewYork City. Antimicrob Agents Chemother. 2015; 59:5029-31.

69. Sims M, Mariyanovski V, McLeroth P, et al. Prospective, randomized, double-blind, Phase 2 dose-ranging study comparing efficacy and safety of imipenem/cilastatin plus relebactam with imipen$\mathrm{em} / \mathrm{cilastatin}$ alone in patients with complicated urinary tract infections. J Antimicrob Chemother. 2017; 72:2616-2626.

70. Zhanel GG, Lawrence CK, Adam $H$, et al. ImipenemRelebactam and Meropenem-Vaborbactam: Two Novel Carbapenem- $\beta$-Lactamase Inhibitor Combinations. Drugs. 2018; 78:65-98

71. Castanheira M, Huband MD, Mendes RE, et al. MeropenemVaborbactam Tested against Contemporary Gram-Negative Isolates Collected Worldwide during 2014, Including Carbapenem-
Resistant, KPC-Producing, Multidrug-Resistant, and Extensively Drug-Resistant Enterobacteriaceae. Antimicrob Agents Chemother. 2017; 61 .

72. Walsh TJ, Bhowmick T, Darouiche R, et al. MeropenemVaborbactam vs. Piperacillin-Tazobactam in TANGO I (a Phase 3 Randomized, Double-blind Trial): Outcomes by Baseline MIC in Adults with Complicated Urinary Tract Infections or Acute Pyelonephritis. Open Forum Inf Dis. 2017; 4:suppl-1:S536.

73. http://www. themedicinescompany.com/investors/news/medicines-company-announces-tango-2-trial-meropenem-vaborbactamformerly-carbavance.

74. Tängdén T, Giske CG. Global dissemination of extensively drugresistant carbapenemase-producing Enterobacteriaceae: clinical perspectives on detection, treatment and infection control. J Intern Med. 2015; 277:501-12.

75. Tascini C, Tagliaferri E, Giani T, et al. Synergistic activity of colistin plus rifampin against colistin-resistant KPC-producing Klebsiella pneumoniae. Antimicrob Agents Chemother. 2013; 57:3990-3

76. Luque S, Escaño C, Sorli L, et al. Urinary Concentrations of Colistimethate and Formed Colistin after Intravenous Administration in Patients with Multidrug-Resistant GramNegative Bacterial Infections. Antimicrob Agents Chemother. 2017; 61 .

77. Zhanel GG, Lawson CD, Zelenitsky S, et al. Comparison of the next-generation aminoglycoside plazomicin to gentamicin, tobramycin and amikacin. Expert Rev Anti Infect Ther. 2012; 10:459-73

78. Livermore DM, Mushtaq S, Warner M, et al. Activity of aminoglycosides, including ACHN-490, against carbapenemresistant Enterobacteriaceae isolates. J Antimicrob Chemother. $2011 ; 66: 48-53$

79. Galani I, Souli M, Daikos GL, et al. Activity of plazomicin (ACHN-490) against MDR clinical isolates of Klebsiella pneumoniae, Escherichia coli, and Enterobacter spp. from Athens, Greece. J Chemother. 2012; 24:191-4.

80. Rodríguez-Avial I, Pena I, Picazo JJ, et al. In vitro activity of the next-generation aminoglycoside plazomicin alone and in combination with colistin, meropenem, fosfomycin or tigecycline against carbapenemase-producing Enterobacteriaceae strains. Int J Antimicrob Agents. 2015; 46:616-21.

81. Connolly LE, Riddle V, Cebrik D, et al. A Multicenter, Randomized, Double-Blind, Phase 2 Study of the Efficacy and Safety of Plazomicin Compared with Levofloxacin in the Treatment of Complicated Urinary Tract Infection and Acute Pyelonephritis. Antimicrob Agents Chemother. 2018; 62.

82. Peirano G, Ahmed-Bentley J, Woodford N, et al. New Delhi metallo-beta-lactamase from traveler returning to Canada. Emerg Infect Dis. 2011; 17:242-4.

83. Navarro-San Francisco C, Mora-Rillo M, Romero-Gómez MP, et al. Bacteraemia due to OXA-48-carbapenemase-producing Enterobacteriaceae: a major clinical challenge. Clin Microbiol Infect. 2013; 19:E72-9.

84. Karageorgopoulos DE, Miriagou V, Tzouvelekis LS, et al. Emergence of resistance to fosfomycin used as adjunct therapy in KPC Klebsiella pneumoniae bacteraemia: report of three cases. J Antimicrob Chemother. 2012; 67:2777-9.

85. Perez F, El Chakhtoura NG, Papp-Wallace KM, et al. Treatment options for infections caused by carbapenem-resistant Enterobacteriaceae: can we apply "precision medicine" to 
antimicrobial chemotherapy? Expert Opin Pharmacother. 2016; 17:761-81.

86. Michalopoulos A, Virtzili S, Rafailidis $P$, et al. Intravenous fosfomycin for the treatment of nosocomial infections caused by carbapenem-resistant Klebsiella pneumoniae in critically ill patients: a prospective evaluation. Clin Microbiol Infect. 2010; 16:184-6.

87. Mendes RE, Castanheira M, Farrell DJ, et al. Longitudinal (2001-14) analysis of enterococci and VRE causing invasive infections in European and US hospitals, including a contemporary (2010-13) analysis of oritavancin in vitro potency. I Antimicrob Chemother. 2016; 71:3453-3458.

88. Yang S, Clayton SR, Zechiedrich EL. Relative contributions of the AcrAB, MdfA and NorE efflux pumps to quinolone resistance in Escherichia coli. J Antimicrob Chemother. 2003; 51:545-556.

89. Stein GE, Schooley SL, Nicolau DP. Urinary bactericidal activ- ity of single doses $(250,500,750$ and $1000 \mathrm{mg}$ ) of levofloxacin against fluoroquinolone-resistant strains of Escherichia coli. Int J Antimicrob Agents. 2008; 32:320-5.

90. Robicsek A, Strahilevitz J, Jacoby GA, et al. Fluoroquinolonemodifying enzyme: a new adaptation of a common aminoglycoside acetyltransferase. Nat Med. 2006;12:83-8.

91. Cattoir, V; Nordmann, P. Plasmid-Mediated Quinolone Resistance in Gram-Negative Bacterial Species: An Update. Curr Med Chem. 2009; 16:1028-1046.

92. van Duin D, Cober ED, Richter SS, et al. Tigecycline therapy for carbapenem-resistant Klebsiella pneumoniae (CRKP) bacteriuria leads to tigecycline resistance. Clin Microbiol Infect. 2014; 20:01117-20.

93. Kim S, Thiessen PA, Bolton EE, et al. PubChem Substance and Compound databases. Nucleic Acids Res. 2016; 44:D1202-13.

\section{Correspondence}

Gianpaolo Perletti, PhD (Corresponding Author)

gianpaolo.perletti@uninsubria.it

Department of Biotechnology and Life Sciences, Section of Medical and Surgical Sciences, Università degli Studi dell'Insubria

Via A. Da Giussano, 12 - 21052 Busto A., Italy

Vittorio Magri, MD

Urology Secondary Care Clinic, ASST-Nord, Milan, Italy

Tommaso Cai, MD

Department of Urology, Santa Chiara Regional Hospital, Trento, Italy

Konstantinos Stamatiou, MD

Department of Urology, Tzaneio General Hospital of Piraeus, Piraeus, Greece

Alberto Trinchieri, $M D$

Urology Unit, A. Manzoni Hospital, Lecco, Italy

Emanuele Montanari, MD

Department of Urology, University of Milan-Ca' Granda Foundation

Ospedale Maggiore Policlinico, Milan, Italy 